\title{
The Speech Signal Noise Elimination based on the Wavelet Packet Transformation in the OFDM Underwater Acoustic Communication System
}

\author{
Yang Lei \\ Wuhan Polytheistic University \\ yl975210@sina.com
}

\begin{abstract}
A method is promoted to eliminate the noise of the speech signal in the orthogonal frequency division multiplexing (OFDM) underwater acoustic communication system based on the wavelet packet decomposition and reconstruction. The wavelet packet decomposition is accomplished by analyzing the different wavelet transform coefficient distribution characteristics of the signal and the noise. The speech signal is stratified to determine the best wavelet packet decomposition tree, the threshold value is quantified, and the wavelet packet decomposition is completed. The threshold value can be used to eliminate signal noise. Finally, the transmitted signal is reconstructed by the inverse transformation of the wavelet packet. The computer simulation results show that the method of wavelet packet decomposition is worked for the speech signal processing in the OFDM underwater acoustic communication system. For the speech signal transferred in the underwater acoustic channel, the noise can be eliminated and the valid signal can be kept effectively.
\end{abstract}

Keywords: Underwater acoustic communication, wavelet packet, OFDM; noise elimination

\section{Introduction}

Underwater acoustic channel is a kind of random channel that the space and the time are varying. High speed, stable speech signal transmission can be realized by overcoming the serious time-frequency dual extension effect in the multipath underwater acoustic channel. This is the key to complete communication tasks in a complex underwater channel. For the high quality, fast speech signal transmission in the underwater acoustic channel, in this paper, the orthogonal frequency division multiplexing (OFDM) technique is applied to the underwater acoustic communication system. OFDM technique can be used to against frequency selective fading effectively. It has some advantages such as the good ability to resist intersymbol interference caused by multipath, good decay resistance ability and high frequency utilization, etc [1]. The underwater acoustic communication system with the OFDM technique has the characteristics of high transmission capacity, stable data transmission. So the system is a hot spot in the research of underwater acoustic communication system. This paper focus on the speech signal processing of de-noising and refactoring using the wavelet packet decomposition for the OFDM underwater acoustic communication system. In this paper, an OFDM underwater acoustic communication system is constructed to transfer the speech signal, the method of wavelet packet decomposition (WPD) is used in this system's receiver to deal with the received signal for a better bit error rate. In this process, the threshold value can be determined by the different distribution characteristics of the signal and the noise wavelet transform coefficient. So the signal de-noising is realized. After that, the signal is reconstructed using the inverse transformation of wavelet packet (WPR). The computer simulation results show that 
this method is effective for the speech signal de-noising processing in the underwater acoustic channel.

\section{The Principle of De-noising OFDM Underwater Acoustic Communication System}

\subsection{The Principle of OFDM}

Fig. 1 shows the OFDM underwater acoustic transmission system. In this system, the underwater acoustic channel is divided into subchannels. The subchannels are orthogonal to each other, and the spectrums are overlapping. This kind of structure can reduce the mutual interference between the subcarriers and improve the spectrum utilization. It is not a flat one to the channel frequency response curve of underwater communication. So the OFDM technology is a kind of high speed transmission technology suitable for the underwater communication system [2].

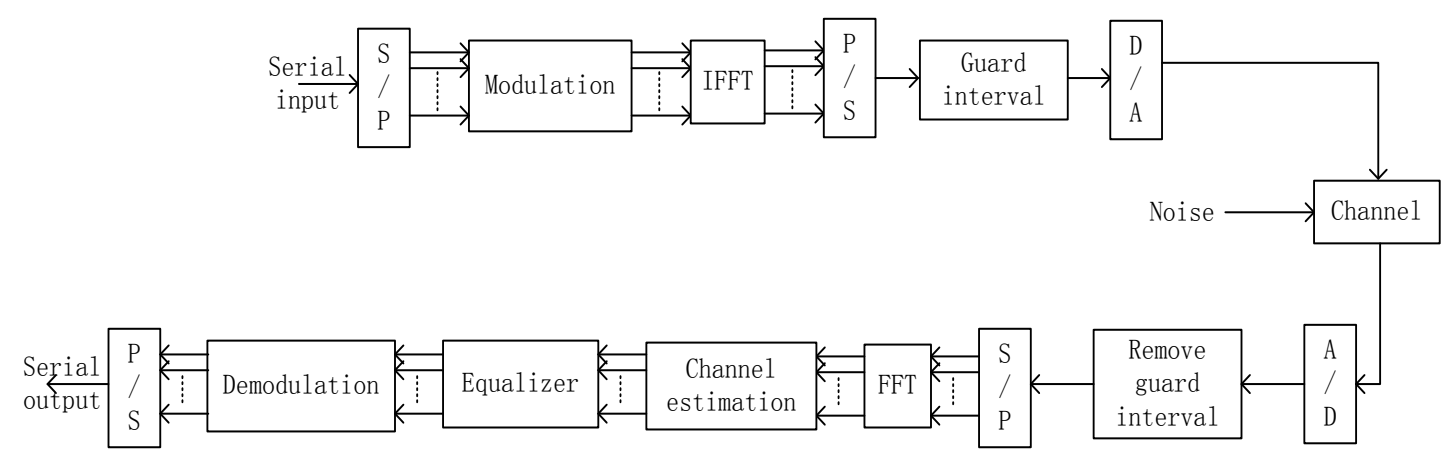

Figure 1. Block Diagram of the OFDM System

On the transmitting terminal, the speech signal serial is inputted, the serial data converted into $\mathrm{N}$ parallel sub-data string symbol by the serial-to-parallel conversion. The sub-stream data length $\mathrm{N}$ times the original length. The bandwidth of sub-data stream is narrower than the original data stream. This kind of mode can resist the delay spread and frequency selective fading effect. An OFDM symbol can be achieved by PSK or QAM modulation of parallel sub-data string. In practice, it can be realized by the general Inverse Fast Fourier Transform or Fast Fourier Transform (IFFT / FFT). Usually the cyclic prefix (CP) is used to overcome the inter-symbol interference (ISI), it can be added between each OFDM symbol. The receiving terminal does the exact opposite process of the transmitting terminal. In this case, the signal we got need to equalized, decoding and other dispose, and finally obtain the final data by a parallel-toserial conversion, thus completing the whole process of communication. Wavelet packet on the signal processing is used in the receiving terminal of OFDM system.

\subsection{The Principle of Wavelet Packet}

The wavelet package transformation can decompose the multi-resolution space to the low-resolution space and the detail space. In other words, the wavelet package transformation can decompose the scale space and wavelet space synchronously. Hence, it avoid the defect that low time resolution and high frequency resolution at high-band, high-time resolution and low frequency resolution at low-band of wavelet 
transformation. This shows that wavelet packet transformation is the analysis method more elaborate than wavelet transformation. There is a decomposition sample figure of three-layer wavelet packet transformation to illustrates the decomposition process, showed in Fig.1.In this figure, $\mathrm{L}$ is used to express low frequency, $\mathrm{H}$ is used to express high frequency, and the number fellows alphabet is used to express the decomposition layer number (scale number).The decomposition relationship can be represented by:

$$
d_{n}=H H H 3+L H H 3+H L H 3+L L H 3+H H L 3+L H L 3+H L L 3+L L L 3
$$

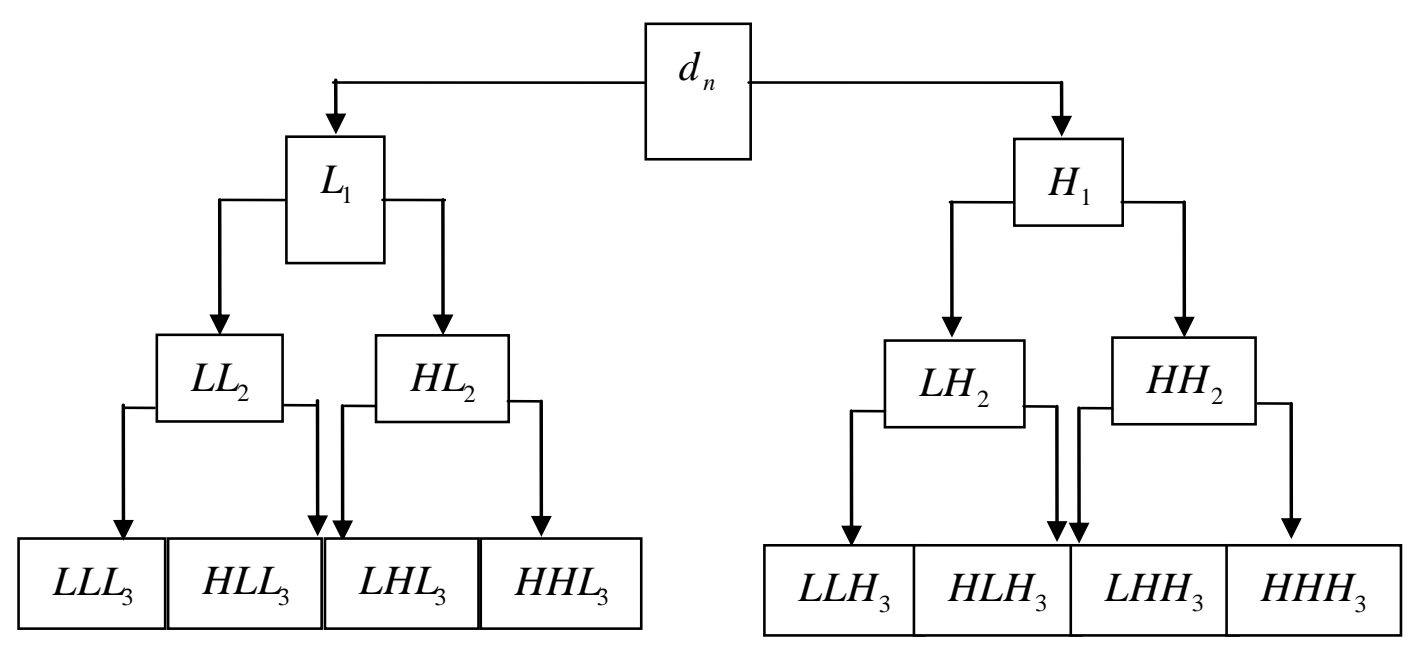

Figure 2. The Schematic Diagram of Wavelet Packet Decomposition (Three Layers)

\subsection{The Principle of the Method Mentioned in this Paper}

The wavelet packet decomposition for signal processing is applied in the receiver of the OFDM system. Figure 3 shows the block diagram of the underwater acoustic communication OFDM system mentioned in this paper.

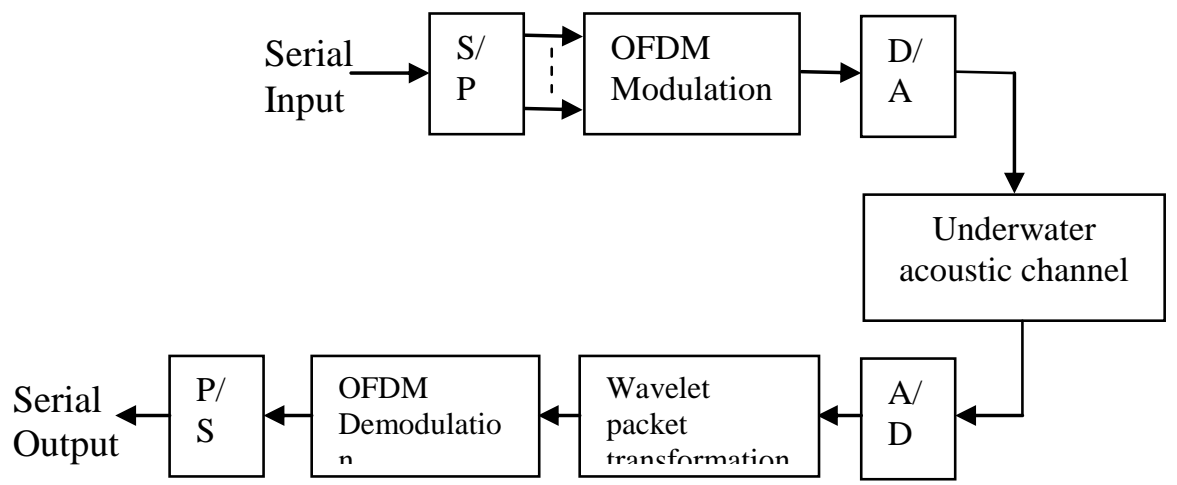

\section{Figure 3. Block Diagram of the Underwater Acoustic Communication OFDM System}

Wavelet packet transform is a sort of signal processing method with high pass filter and low pass filter continuously, the wavelet packet transform can be implemented by using quadrature mirror filter (QMF) group for signal decomposition and reconstruction of a uniform or non-uniform binary tree structure, the time-frequency units of the timefrequency diagram correspond to the nodes in the tree structure, and corresponds to the particular subbands from the point of view of filter banks [3]. When the node forward decomposition (reverse merger) cause the number of the time-frequency unit 
interference increase, the reverse merger (forward decomposition) will not reduce the influence of interference; When the node forward decomposition (reverse merger) cause the number of the time-frequency unit interference reduce, the reverse merger (prior to decomposition) will increase the influence of interference. The problem of tracking and positioning interference accurately is converted to the problem of establishing the optimal wavelet packet binary tree [4]. To find the best wavelet packet tree structure, a judgment must be able to make when the interfere component has been concentrated in the least amount of time-frequency unit, and then the forward merge or reverse merger can stop, namely the measurement need to be found to measure the energy concentration of interference. Here the parameter of subband coding gain SCG (sub band code gain) is for reference mainly. The $\mathrm{M}$ subband coding gain is defined as [5],

$$
S C G=\frac{\sigma_{x}^{2}}{\left[\prod_{i=1}^{M} \sigma_{i}^{2}\right]^{1 / M}}
$$

In which, $\sigma_{x}^{2}$ is the variance of input signal, $\sigma_{i}^{2}$ is the variance of $i$ output signal.

The energy concentration can be expressed by G,

$$
G=\frac{\sigma^{2}}{\sigma_{l} \sigma_{r}}
$$

In which, $\sigma^{2}$ is the coefficient variance of father node, $\sigma_{l}$ and $\sigma_{r}$ are the standard deviation of child node.

In the wavelet packet decomposition, a reference threshold should be set in advance, when the energy concentration is greater than the threshold value, the corresponding subband should continue to decompose, and when the energy concentration is less than the threshold value, the corresponding subband should reverse merger, and then the optimal decomposition binary tree is found finally. The location of the interference is also determined, the accurate tracking and positioning of narrow-band interference is completed. Like the narrowband interference suppression algorithm that based on FFT, there are two ways for the interference wavelet coefficient, zero setting directly and adaptive filtering.

In this paper, adaptive filtering is used to find the optimal decomposition binary tree to deal with the corresponding subband signal data, thus achieving the purpose of interference suppression. If the maximum resolution is $\pi / 2^{M}$ (M is the maximum depth of wavelet packet decomposition binary tree), the precise steps of narrow band interference restrain are,

(1) Wavelet packet decomposition process:

If the sampling rate based on PN sequence, the input signal can be decomposed by wavelet packet. The D depth of wavelet packet decomposition binary tree can be obtained. We can calculate the standard deviation $\sigma_{D-1, i}$ of ith node with (D-1) depth and the corresponding standard deviation $\sigma_{D, 2 i}$ and $\sigma_{D, 2 i+1}$ of the other two child nodes. The energy concentration $G_{D-1, i}$ of ith node is calculated by expression (2). Compared with $G_{D-1, i}$ and the given threshold $T_{D-1}$, if $G_{D-1, i}>T_{D-1}$, there is noise interference at the father node, so do the forward decomposition to the two child nodes, and continue to compare the energy concentration, repeat the steps until $G_{d, i}<T_{d}$ or to attach the maximum depth. If $G_{d, i}<T_{d}$, the frequency band of father node is composed of the 
noise interference or the component without noise interference, so reverse merger to node until $G_{d, i}>T_{d}$ or to attach the depth equal to 1 .

(2)De-noising process

A parameter $\eta=\sigma_{l} / \sigma_{r}$ is set to stand for the subband energy ratio. All the subnodes of irregular binary tree will be got with the step (1). When the energy concentration of father node is bigger than the threshold, and the energy concentration of two subnodes is smaller than the threshold, the father node can be set to zero directly. Otherwise, if $\eta>T_{\eta}$ ( $T_{\eta}$ is the subband signal value that subtract threshold), so the left node is composed by noise interference, the coefficient of corresponding subband can be set to zero. if $\eta<T_{\eta}$, the right node is composed by noise interference, the coefficient of corresponding subband can be set to zero.

(3) Reconstruct the speech signal without noise by wavelet packet inverse transformation.

\section{Channel Model}

When the acoustic signal transmitted underwater, the multipath effect is a major cause of underwater acoustic signal distortion. Underwater acoustic channel is a typical time-varying multi-path fading channel. The received signal can be seen as a superimposed signal got from different paths.

If the ocean-related multi-path channel impulse response function is $h(\tau)$ :

$$
h(\tau)=a_{0} \delta\left(\tau-\tau_{0}\right)+\sum_{i=1}^{N-1} a_{i}\left(\tau-\tau_{i}\right)
$$

In which, $a_{0}$ is the voice amplitude parameters of the receiving point, $\tau_{i}$ is the voice delay parameter of the receiving point, $\mathrm{N}$ is the number of resolvable multipath components.

And if the acoustic emission line signal is $s(t)$, the received waveform at the receiving terminal is :

$$
r(t)=a_{0} s\left(t-\tau_{0}\right)+\sum_{i=1}^{N-1} s\left(\tau-\tau_{i}\right)+n(t)
$$

In which, $a_{0} s\left(t-\tau_{0}\right)$ is the direct sound, $\sum_{i=1}^{N-1} s\left(\tau-\tau_{i}\right)$ is the multi-way extension, Multi-way extension overlap with the direct sound in time, and also produce interference, so that the waveform signal is different from the transmitted signal because of the distortion.

A kind of underwater acoustic channel models can be expressed as [6]:

$$
h(\tau)=a_{0} \delta\left(\tau-\tau_{0}\right)+\sum_{i=1}^{N-1} a_{i}\left(\tau-\tau_{i}\right)
$$

In which, $a_{0}$ is the acoustic amplitude parameters of receiving point, $\tau_{i}$ the acoustic time delay parameters of receiving point, $\mathrm{N}$ is the number of multipath.

There is a three path underwater acoustic channel. The time delay and the attenuation coefficient are $0 \mathrm{~ms}$ and 1 in the first path, and $5 \mathrm{~ms}$ and 0.3 in the second path, and 10 ms and 0.1 in the third path.

\section{Simulations}

We simulated the method based on the wavelet packet decomposition for denoising speech signal transmission in the OFDM underwater acoustic 
communication system. Here are the simulation parameter settings: the center frequency is $25 \mathrm{kHz}$, subband width is $20 \mathrm{~Hz}$, period is $50 \mathrm{~ms}$ and cyclic prefix is $10 \mathrm{~ms}$, subcarrier number is 512, IFFT/FFT number is 1024 , Modulation mode is BPSK modulation. The channel model is the three path underwater acoustic channel model described above. SNR range is $-5 \mathrm{~dB}-15 \mathrm{~dB}$. The synchronization of the underwater acoustic system is the ideal synchronization. The channel estimation algorithm is the LS algorithm estimation [7][8] and one-dimensional MMSE algorithm [9][10]. According to the principle of wavelet packet de-noising discussed above, two algorithm of OFDM channel estimation (LS and onedimensional MMSE) is used to eliminate speech signal noise interference in the underwater acoustic communication system.

Figure 4 shows the initial speech signal, Figure 5 shows the speech signal with noise, Figure 6 shows the speech signal after eliminated noise by wavelet package transformation with LS channel estimation. From the Fig.4, it can be seen that most of the noise in speech signal is eliminated, and the available information is kept mainly.

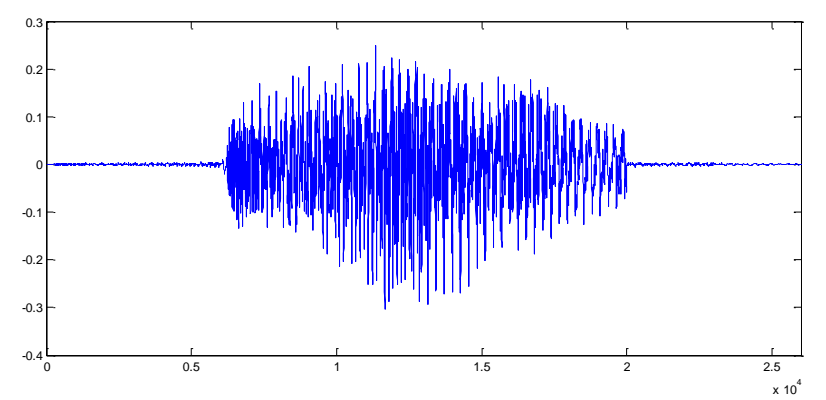

Figure 4. The Schematic Diagram of Initial Speech Signal

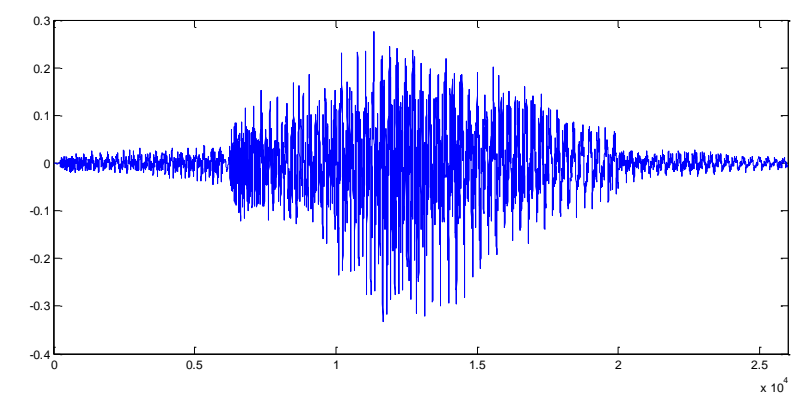

Figure 5. The Schematic Diagram of Initial Speech Signal with Noise

Figure 7 is the comparison curves that the de-nosing signal bit error rate based on wavelet packet decomposition with LS algorithm channel estimation and with one-dimensional MMSE algorithm channel estimation. The four curves from top to bottom separately show that, the first one is the signal transmission BER curve of the OFDM underwater acoustic communication with LS algorithm channel estimation, the second one is the BER curve with LS algorithm channel estimation and wavelet packet decomposition, the third one is the BER curve with onedimensional MMSE algorithm channel estimation, the fourth one is the BER curve with one-dimensional MMSE algorithm channel estimation and wavelet packet decomposition. 


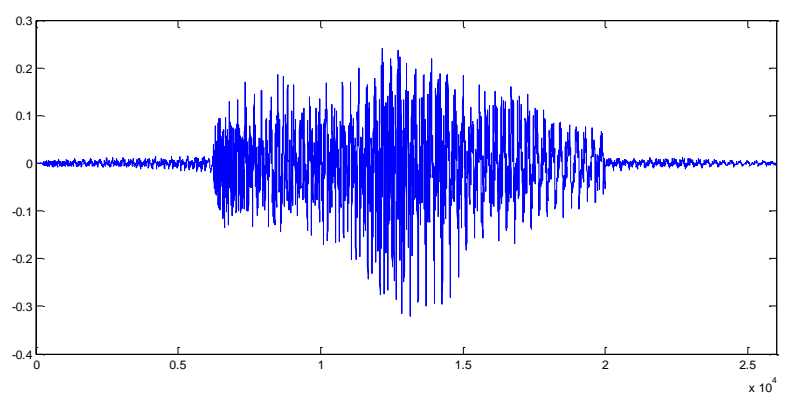

Figure 6. The Schematic Diagram of Speech Signal after Eliminated Noise by Wavelet Package Transformation

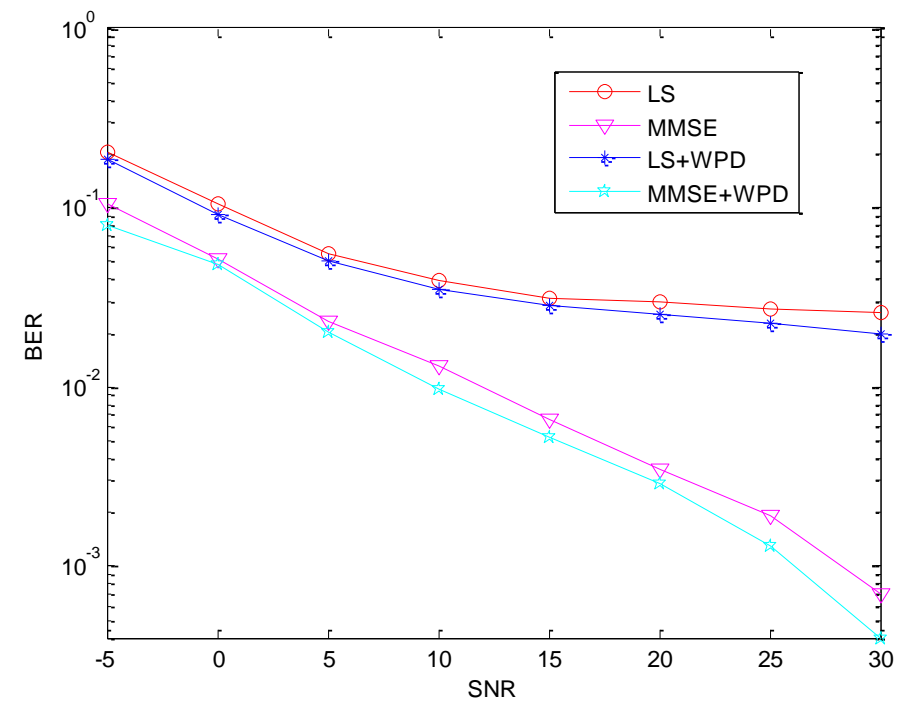

\section{Figure 7. Comparison Diagram of the Underwater Acoustic Communication OFDM System with Wavelet Packet Transformation}

The conclusion can be reached from the figures, when using different channel estimation algorithm, wavelet packet de-noising method is effective. With the same channel estimation algorithm, the error rate with the wavelet packet decomposition better than that of without decomposition obviously, and with the increase of signal-to-noise ratio, this trend is more obvious. This shows that it is useful to the speech signal de-nosing in OFDM underwater acoustic communication system with wavelet packet decomposition treatment.

\section{Conclusion}

A kind of speech signal noise elimination method is proposed that utlize wavelet packet transformation. This kind of method can work well in the underwater acoustic communication system. It can eliminate speech signal noise by analyze threshold value effectively.It is found that most of the noise can be eliminated and the useful signal can be fully kept in the underwater acoustic communication. It means that this method is efficient in speech signal noise elimination in the system. 


\section{Acknowledgements}

Science and technology research planning projects of Hubei Provincial Education Department. The project number is B20121803.

\section{References}

[1] W. Wenbo, Z. Kan and X. Henghua, "OFDM Broadband Wireless Communication Technology", Posts and Telecom Press, Beijing, (2003).

[2] T. Xuejian, L. Tao and C. Wanshou, "Principles and Applications of OFDM mobile communication technology", Posts and Telecom Press, Beijing, (2003).

[3] L. K. Jiwani, S. D. Joshi SD, G. S. Visweswaran, "Priority Wavelet Packet Decomposition and Representation”, IET Signal Processing, vol. 6, no. 5, (2011).

[4] H. N. Jagannath, A. Z. Mukesh, P. Suprava and H. K. Pramod, "A novel voice conversion approach using admissiblewavelet packet decomposition”, EURASIP Journal on Audio, Speech, and Music Processing, vol. 1, (2013).

[5] C. Yunpeng, Z. Kaiyuan and X. Zhong and L. Jun, "Matrix theory", Northwestern Polytechnic University Press, Xian, (2006).

[6] H. Junying, S. Xueli and Z. Linfeng, "Underwater Acoustic Channel", Harbin Engineering University Press, Harbin, (2011).

[7] M. Bogdanovic, "Frequency domain based LS channel estimation in OFDM based Power line communications", Science Citation Index Expanded, vol. 4, no. 55, (2014).

[8] Y. Linchao, T. Ricai, J. Min and L. Feng, “A Modified LS Channel Estimation Algorithm for OFDM System in Mountain Wireless Environment”, Procedia Engineering, vol. 29, (2012).

[9] S. R. Pierluigi, R. M. Ralf, E. Ove, "Linear MMSE Estimation of Time-frequency Variant Channels for MIMO-OFDM Systems. Signal Processing”, vol. 5, no. 91, (2011).

[10] H. M. Kim, D. Kim, T. K. Kim, G. H. Im, "Frequency Domain Channel Estimation for MIMO SC-FDMA Systems with CDM Pilots", Journal of Communications and Networks, vol. 4, no. 16, (2014). 